\title{
Folk medicine used to heal malaria in Calabria (southern Italy)
}

\author{
Giuseppe Tagarelli, Antonio Tagarelli, Anna Piro
}

\begin{abstract}
In Italy, malaria was an endemic disease that was eradicated by the mid-20th century. This paper evaluates the prophylactic and therapeutic remedies used by folk medicine to cure malaria in Calabria (southern Italy). The data has been collected by analysing works of physicians, ethnographers, folklorists and specialists of the study of Calabrian history between the end of the 19th century and the 20th century. The data collected have allowed us to describe the most common cures used by the Calabrian people to treat malaria and the most evident symptoms of this disease, such as intermittent fever, hepato-spleenomegaly, asthenia and dropsy. This approach uncovered a heterogeneous corpus of empirical, magical and religious remedies, which the authors have investigated as evidences of past "expert medicine" and to verify their real effectiveness in the treatment of malaria.
\end{abstract}

\section{Background}

Malaria is an infectious disease that is caused by the Plasmodium parasite. This disease is transmitted to humans via the Anopheles mosquito. Malaria is a very ancient disease, and although it was not possible to prove its presence in ancient human bones, this disease was probably present among Homo genus ancestors [1]. Different populations, such as the Sumerians, AssyrianBabylonians, Indians, Egyptians and Chinese, experienced seasonal and intermittent fevers [2]. In the Mediterranean area, particularly in Italy, malaria was an endemic disease that was eradicated by the mid-20th century. Moreover, the persistent and lasting presence of malaria determined an interesting state of debility of the affected subjects and a consequent weakening of the labour force, which led to some important and detrimental socio-economic consequences [3]. Folk medicine approaches were used in an attempt to treat several of the most evident effects of malaria, such as intermittent fever, hepato-spleenomegaly, asthenia and dropsy.

It is our aim in this work to identify folk medical cures that were used by the Calabrian people for the treatment of malaria, as evidenced in writings produced between the 19th and 20th centuries. The authors have also examined whether same remedies were already described by Pliny the Elder, Dioscorides, Galen and

\footnotetext{
* Correspondence: g.tagarelli@isn.cnr.it

Istituto di Scienze Neurologiche-CNR, Contrada Burga 87050 Mangone, Cosenza, Italy
}

Serenus Sammonicus, so to be considered as evidence of past "expert medicine".

\section{Area of Study}

It is interesting to point out that in some peninsular and insular areas of Italy, despite all the drainage attempts initiated in the 16th century, malaria-associated mortality was only recently eradicated in the mid-20th century (Figure 1) [4]. Among the southern regions of Italy, Calabria was one of the regions that was most affected by malaria. The disease was endemic along its coasts (about $738 \mathrm{~km}$ ), along its most important rivers (Mesima, Lao, Crati, Tacina and Neto) and within the valleys of its broad streams. The disease was prevalent in $52 \%$ of the Calabrian territory $(7,877.31 / 15,080.32$ $\mathrm{km}^{2}$ ) (Figure 2) [5]. Calabria showed both natural and antropic factors that favoured the spread of Plasmodium, as well as the endemic and century-old presence of malaria in its territory. Physical features that may have affected the spreading of malaria are represented by a rich hydrographic reticle and the occurrence of seismic phenomena (bradyseisms and earthquakes), which, at that time, contributed to increase the hydrogeological disorder, thus creating many different wet areas (for example, the single earthquake of 1783 created about 215 lakes), which are the favourite environment of the anopheles mosquito [6]. The antropic factors are represented fundamentally by latifundia, 


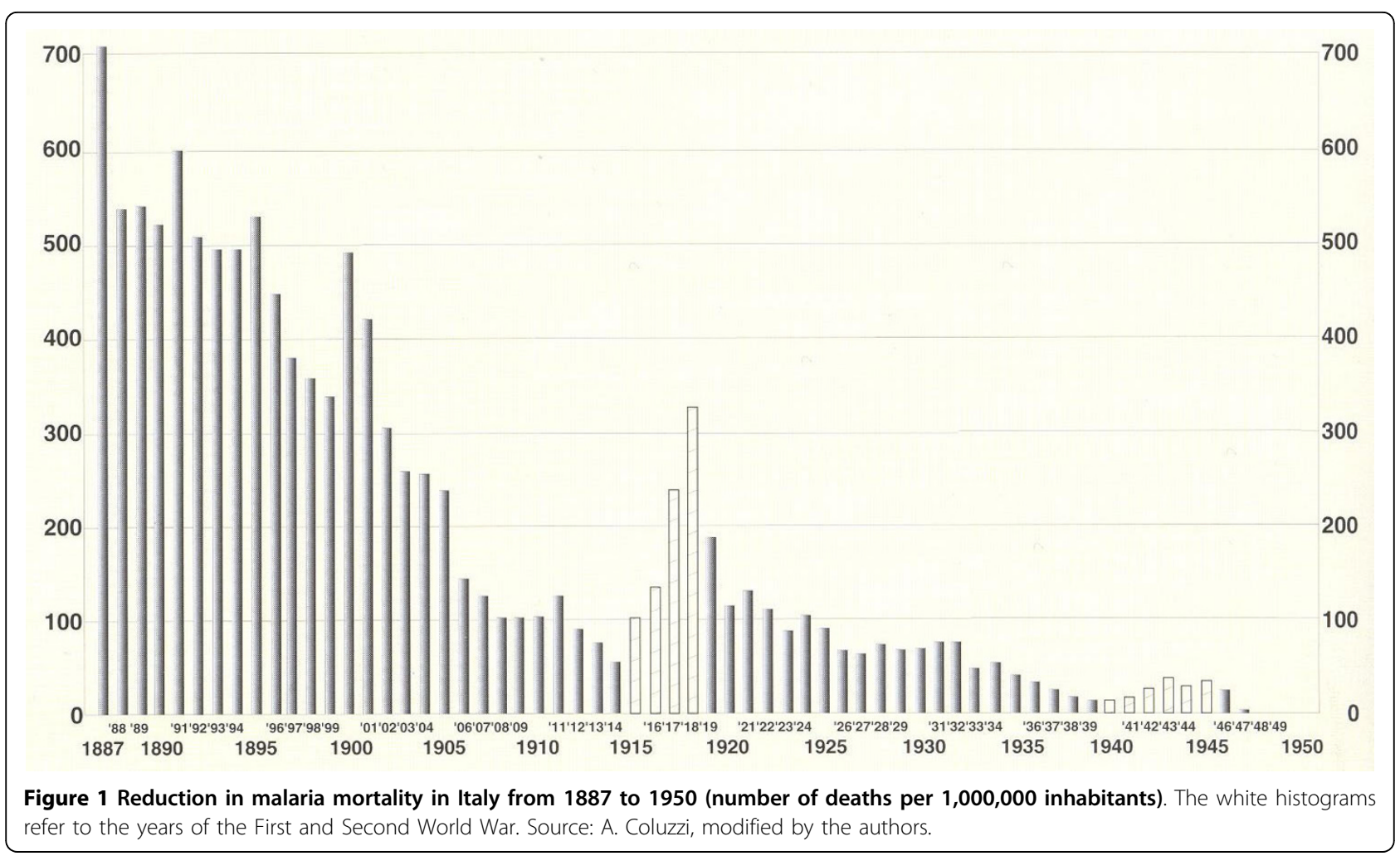

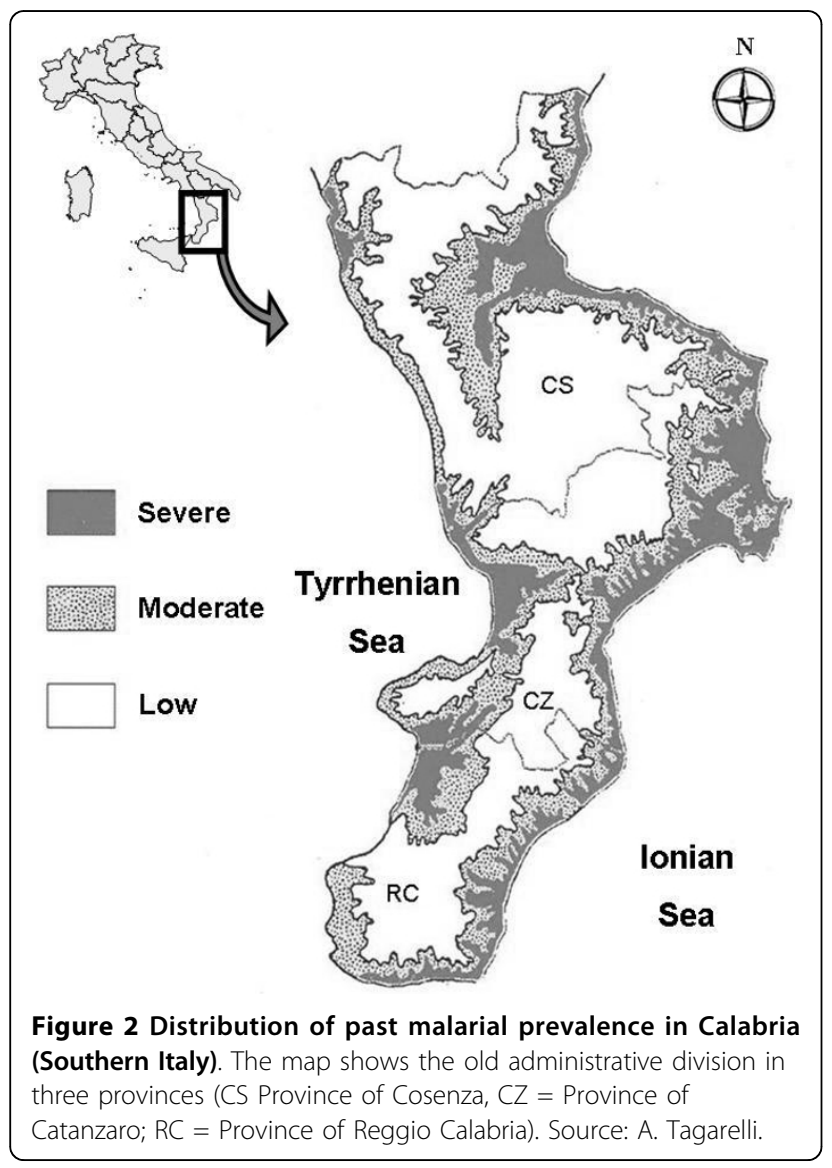

deforestation and the very poor social and economic conditions of the rural Calabrian people [7].

Calabria is the southernmost region of peninsular Italy; it borders with the Ionian Sea to the east and south, with the Tyrrhenian Sea to the west, and with the region Basilicata to the north, and it extends for about $250 \mathrm{~km}$ from north to south in the middle of the Mediterranean Sea. Calabria reaches $15,080 \mathrm{~km}^{2}$ and $42 \%$ of its territory is represented by mountains: the Apennine mountain range - the southern Apennines, calcareous, with Pollino Massif (Serra Dolcedorme, $2267 \mathrm{~m}$ a.s.l.)- and the Calabrian Apennines, mainly siliceous - with the Coastal Range (M. Cocuzzo,1541 m a.s.l.), Sila Massif (M. Botte Donato,1929 m a.s.l.), Serre Calabre (M. Pecoraro, 1423 m a.s.l.) and Aspromonte Massif (Montalto, $1956 \mathrm{~m}$ a.s. 1.)-. $49 \%$ of Calabria's territory is represented by hills and only $9 \%$ is flat. The plains are restricted to coastal areas and only three plains extend into the internal areas of the territory (Sibari plain, Saint'Eufemia plain and Gioia Tauro plain).

The climate is Mediterranean, with maximum precipitation during the winter and minimum in the summer and vice versa for the temperature. Precipitation is represented by about $1,041 \mathrm{~mm}$ of rainfall per year. The average temperature in the coldest month (January) is about $8.3^{\circ} \mathrm{C}$ and the warmest month (August) about $24.4^{\circ} \mathrm{C}$, with an annual average of $15.8^{\circ} \mathrm{C}$ [8]. However strong meso-climatic variations occur depending on 
altitude, topographic features and location with respect to the sea.

From both an anthropological and an ethnobotanical point of view, Calabria is an interesting region, for the historical presence of several populations (Greeks, Romans, Byzantines, Arabs, Normans and Spanish) in the past that influenced the local culture [9]. Finally, it is interesting to note that the Arbëreshe community, of Albanian origins, settled in this region since the 16th century and is currently located in 25 communes in the provinces of Cosenza, Catanzaro and Crotone [10].

\section{Methods}

This study is based on the analysis of works written by physicians, ethnographers, folklorists and specialists of the study of Calabrian history between the end of the 19th century and the 20th century, in particular when both ethnographic and anthropological research focused on the study of folk medicine. For this reason, the authors have also consulted the check-lists about works on calabrian folk medicine and beliefs, edited by Lombardi Satriani [11] and Cavalcanti [12]. The sources used in this work are listed in Table 1. The data collected has allowed us to describe the cures used by the Calabrian people to treat malaria and its most evident symptoms, such as intermittent fever, hepato-spleenomegaly, asthenia and dropsy. This approach uncovered a heterogeneous corpus of empirical, magical and religious remedies, which the authors have investigated as an "official medicine" to cure malaria in the treatises by Pliny the Elder (Naturalis Historia) [13-15], Dioscorides (De Materia Medica) [16], Galen (Opera Omnia) [17-21] and by Serenus Sammonicus (Liber Medicinalis) [22]. These authors, in fact, have influenced medical practice in latter centuries [23].

The plants used by Calabrian people to cure malaria $(\mathrm{N}=53)$ have been identified as species because the

Table 1 Sources used for the research

\begin{tabular}{|c|c|c|c|}
\hline Author & Period $^{\circ}$ & Description & Reference \\
\hline $\begin{array}{l}\text { Francesco } \\
\text { Genovese }\end{array}$ & $\begin{array}{l}1912- \\
1924\end{array}$ & Physician, malariologist who wrote about malaria in Calabria. & {$[6,56,62]$} \\
\hline Alessandro Adriano & 1932 & Physician whose daily experiences provided information about folk Calabrian medicine. & {$[65]$} \\
\hline $\begin{array}{l}\text { Giovanni De } \\
\text { Giacomo }\end{array}$ & $\begin{array}{l}1892- \\
1896\end{array}$ & $\begin{array}{l}\text { Folklorist who published many works about folk culture including therapeutic remedies to cure many } \\
\text { diseases. }\end{array}$ & {$[26,54,55,57]$} \\
\hline $\begin{array}{l}\text { Gianbattista } \\
\text { Marzano }\end{array}$ & $1889-$ & $\begin{array}{l}\text { Folklorist and historian who wrote about folk traditions in Laureana di Borrello (province of Reggio } \\
\text { Calabria). He published a vocabulary of south Calabrian dialect with historical and folkloric notes as well. }\end{array}$ & {$[25,68]$} \\
\hline $\begin{array}{l}\text { Raffaele Lombardi } \\
\text { Satriani }\end{array}$ & $\begin{array}{c}1916- \\
1951\end{array}$ & Folklorist and ethnographer who published many works about the Calabrian people. & {$[11,53]$} \\
\hline Luigi Accattatis & 1895 & $\begin{array}{l}\text { Historian and linguist who published a vocabulary of north Calabrian dialect with historical and folkloric } \\
\text { notes. }\end{array}$ & {$[24]$} \\
\hline $\begin{array}{l}\text { Filippo Jacopo } \\
\text { Pignatari }\end{array}$ & $\begin{array}{l}1894- \\
1895\end{array}$ & $\begin{array}{l}\text { Man of letters who published many papers about Calabrian beliefs and traditions including the use of } \\
\text { plants and animal to cure many diseases. }\end{array}$ & {$[58-60,67,75]$} \\
\hline $\begin{array}{l}\text { Luca De Samuele } \\
\text { Cagnazzi }\end{array}$ & 1811 & Mathematician who edited the statistical report of the Kingdom of Naples of 1811. & {$[72]$} \\
\hline $\begin{array}{l}\text { Vincenzo } \\
\text { Donnarumma }\end{array}$ & 1951 & $\begin{array}{l}\text { Franciscan monk who wrote a book about the religious cult of the Madonna in the province of } \\
\text { Cosenza. }\end{array}$ & {$[78]$} \\
\hline Antonio lannicelli & 1991 & Writer who published a book about Calabrian folk traditions. & {$[79]$} \\
\hline Vincenzo Romeo & 1946 & Physician who published a work about anti-malaria prophylaxis. & {$[74]$} \\
\hline Leopoldo Pagano & $\begin{array}{l}1853- \\
1901\end{array}$ & $\begin{array}{l}\text { Historian and man of letters. He wrote an important book about the economy, history and environment } \\
\text { of Calabria. }\end{array}$ & {$[66,70]$} \\
\hline $\begin{array}{l}\text { Placido Olindo } \\
\text { Geraci }\end{array}$ & 1957 & Man of letters was author of a paper about folk Calabrian medicine. & {$[64]$} \\
\hline Biagio Lanza & $\begin{array}{c}1853- \\
1860\end{array}$ & Physician and author of a paper about folk medicine in Cassano (province of Cosenza). & {$[63]$} \\
\hline Raffaele Corso & 1953 & $\begin{array}{l}\text { Along with Lombardi Satriani, he was one of the most important Italian folklorists and etnhographers. } \\
\text { He wrote many works about amulets. }\end{array}$ & {$[77]$} \\
\hline Vincenzo Brancia & $\begin{array}{c}1853- \\
1860\end{array}$ & Priest who wrote a paper about folk medicine in Nicotera (province of Catanzaro). & {$[69]$} \\
\hline Lorenzo Galasso & 1915 & Priest who wrote a book about the Calabrian people & {$[76]$} \\
\hline Michele Tenore & 1827 & Botanist who published a work on Prunus spinosa L. & {$[73]$} \\
\hline Silvio Mollo & 1930 & Man of letters and author of a book about Calabrian folklore & [71] \\
\hline
\end{tabular}


sources described them with their scientific name $(34 \%$ of cases, 18/53), or with their Italian name $(60 \%$ of cases, 32/53). In the latter case, we attributed a plant to a species only if we were absolutely sure about it (for example "olivo" (olive) = Olea europaea L.); in the other cases we classified the plants only through their genus (for example "quercia" (oak) = Quercus spp.). Furthermore, due to the presence of different dialects in Northern, Central and Southern Calabria, the analysis of the different historical sources required the use of two historical dictionaries of Calabrian dialects [24,25], which were used to translate several ancient terms (that are not used today) into Italian in $4 \%$ of cases $(2 / 53)$. This has allowed us to attribute the local name "vruca" to the genus Tamarix spp., while it has not allowed us (2\% of cases, 1/53) to attribute the local name "catabuzzico" [26] to any genus. Finally, the plants used to cure malaria in Calabria $(\mathrm{N}=52)$ have been compared with their pharmacological and biological properties [27-48] and with their current use in Calabrian folk medicine, defined by recent fieldworks [49-51] [Table 2].

The family names of the plants recorded in this work follow the Angiosperm Phylogeny Group guidelines [52].

\section{Results}

\section{Empirical remedies}

The empirical remedies that were used by the Calabrian people, as evidenced by the sources consulted in this work, were used both prophylactically and therapeutically, and were based on drinks, objects, animals, plants and other sources; some of these elements were created ex novo, while others were inherited from the "official" medicine of 1th-3th century AD [Tables 3 and 4].

Empirical prophylactic remedies. One of the most commonly used prophylactic empirical remedies was bleeding (which was already described by Galen that affirmed "Saluberrimum igitur, ut praediximus, est in febribus venam incidere" (during the fever, as mentioned, it is very useful to incise a vein) (De Methodo Medendi XI, 15) [17]. Bleeding was performed preventively by "barbieri" (barbers) and "magare" (witches) during the month of March [53]. During the same period (when the cure was called "marziale"), Calabrian people drank different types of decoctions, such as those made with "durcamara" (Solanum dulcamara L.), "acropastu" (Cynodon dactylon (L.) Pers.), "strazza buttuni” (Smilax aspera L.) and "fumaria" (Fumaria officinalis L.) [11,54]. To prevent contagion of the disease during the night in the summer months, people slept for few hours and near a fire [54-56]. Moreover, they drank strong spirits or wine. In particular, they were advised to drink half a litre of wine on an empty stomach $[57,58]$, eat garlic (Allium sativum L.) [59], smoke and chew tobacco and swallow the spittle $[56,60]$, while always maintaining the pipe in the mouth [54-56]. On awaking, it was recommended to eat a macerate of raw garlic in vinegar [54]. Finally, people living on the coast used to spread olive oil mixed with absinth on their bodies, according to Dioscorides who affirmed "Itemque ex oleo perunctum, culices abigere, ne corpus tangant" (Rubbed on with oil it forbids the mosquitos to touch the body) De Materia Medica, III, 23) $[16,61]$ and according to Pliny "culices ex oleo perunctis abigit" (who use this oil keep mosquitos away), Naturalis Historia XXVII, 28) [13].

Empirical therapeutical remedies. Fasting and purging were recommended for the treatment of malaria-associated fevers. Fasting was thought to appease fever, while purging was thought to remove the malaria-causing parasite from the affected organism. In general, purging was achieved via the administration of ricinus seeds (Ricinus communis L.) and by using the root of "savucu" (Sambucus nigra L.) [26]. Fasting and purging were inherited from Galenic medicine. Galen wrote the following about fasting: "(in tertiariis) ... neque quotidie cibum dare oportet, sed alternis diebus abunde fuerit" (with tertian fevers ... food must not be offered every day, but on alternate days) (Ad Glauconem de medendi methodo I, 11) [18]; and about purging: "ac vacuatio quidem excrementorum omni febri est utilissima" (during the fever, no doubt, it is very useful to defecate) (Methodo medendi IX, 10) [17]. Other treatments aimed at purging and restoring the affected subjects were also used. These included the decoction of "gamumilla" (Matricaria chamomilla L), "ordica” (Urtica dioica L., Urtica urens L.), and the decoction of the root of "alivu" (Olea europaea L.) or of the rhizome of "canna" (Arundo donax L.) $[26,62,63]$. Several empirical therapeutic remedies against malaria-associated fevers were loathsome. These included the ingestion of the subject's own urine, that of young virgin or that of a healthy woman (in particular, the affected subject was advised to drink $100 \mathrm{~g}$ of the urine of a non-affected woman early in the morning) $[55-57,64]$, the consumption of various animals (or parts of animals), such as earthworms (Lumbricus terrestris) which were previously placed in the oven and pulverized, or two or three bedbugs (Cimex lecturalius) within a Host [56,58,64]. Moreover, patients were encouraged to eat pills of "pappici" (cobweb) [26,56,63-66], the head of a viper (Vipera aspis), fried and mixed with absinth [55], goat (Capra hircus) dung within a Host, eaten from morning until midday $[26,57]$, one spoonful of coffee per hour [57] and pills of soot $[67,68]$. Alternatively, to cure hepato-spleenomegaly were used hedgehog (Erinaceus europaeus) or ox (Bos taurus) gall and goat (Capra hircus) 
Table 2 Medical use of plants to heal malaria in Calabria, bio-pharmacological properties and current use in Calabrian folk medicine

\begin{tabular}{|c|c|c|c|c|c|c|c|c|}
\hline \multicolumn{3}{|c|}{ Plants used to heal malaria in Calabria } & \multicolumn{3}{|c|}{ Current use in Calabrian folk medicine $^{\circ}$} & \multirow{2}{*}{$\begin{array}{c}\text { Pharmacological/ } \\
\text { biological proprierties }\end{array}$} & \multirow{2}{*}{$\begin{array}{l}\text { Used } \\
\text { part }\end{array}$} & \multirow[t]{2}{*}{ Reference } \\
\hline $\begin{array}{c}\text { Family/ } \\
\text { Scientific } \\
\text { Name }\end{array}$ & $\begin{array}{l}\text { Local } \\
\text { Name }\end{array}$ & References & Aim & $\begin{array}{l}\text { Used } \\
\text { part }\end{array}$ & References & & & \\
\hline \multicolumn{9}{|l|}{ Adoxaceae } \\
\hline $\begin{array}{c}\text { Sambucus nigra } \\
\text { L. }\end{array}$ & savucu & {$[26,55]$} & $\begin{array}{c}\text { Antirheumatic, } \\
\text { arteriosclerosis, febrifugal, } \\
\text { purgative, to treat swollen } \\
\text { breast and legs, insect } \\
\text { bites, toothache, colics, } \\
\text { conjunctivitis }\end{array}$ & $\begin{array}{l}\mathrm{Fi}, \mathrm{Fr}, \\
\text { Le, Ba, } \\
\text { Dfh }\end{array}$ & {$[49-51]$} & $\begin{array}{l}\text { Diaphoretic, anti- } \\
\text { inflammatory, diuretic }\end{array}$ & $\mathrm{Fl}, \mathrm{Ba}$ & [27] \\
\hline \multicolumn{9}{|l|}{ Alliaceae } \\
\hline Allium cepa $\mathrm{L}$. & cipuddra & [60] & $\begin{array}{c}\text { To treat flu, cough and } \\
\text { headache, antidiarrhoeic, } \\
\text { vermifugue }\end{array}$ & $\mathrm{Bl}$ & [49] & $\begin{array}{c}\text { Antibacterial, } \\
\text { vermifugue, } \\
\text { hypotensive, diuretic, } \\
\text { hypoglycaemic, eupeptic }\end{array}$ & $\mathrm{Bl}$ & [27] \\
\hline $\begin{array}{c}\text { Allium sativum } \\
\mathrm{L} .\end{array}$ & agliu & {$[55,60]$} & $\begin{array}{l}\text { To treat insect bites, } \\
\text { neuralgias, calluses, } \\
\text { rheumatisms, hypotensive, } \\
\text { tinea, scabies, tooth decay, } \\
\text { cold, diarrhoea }\end{array}$ & $\mathrm{Bl}, \mathrm{Cl}$ & {$[49]$} & $\begin{array}{l}\text { Hypotensive, } \\
\text { antibacterial, } \\
\text { hypoglycaemic, lipid- } \\
\text { lowering, anti- } \\
\text { inflammatory }\end{array}$ & $\mathrm{Bl}$ & [27] \\
\hline \multicolumn{9}{|l|}{ Apocynaceae } \\
\hline $\begin{array}{c}\text { Nerium oleander } \\
\mathrm{L} .\end{array}$ & leandru & [58] & Not Reported & -- & -— & Cardiotonic & Le & [28] \\
\hline \multicolumn{9}{|l|}{ Asteraceae } \\
\hline $\begin{array}{c}\text { Achillea } \\
\text { millefolium L. }\end{array}$ & millefogghio & {$[58,71]$} & Emmenagogue & Ap & [49] & $\begin{array}{c}\text { Eupeptic, cholagogic, } \\
\text { choleretic, antidyspeptic }\end{array}$ & $\mathrm{Fl}, \mathrm{Ap}$ & [27] \\
\hline $\begin{array}{c}\text { Artemisia } \\
\text { absinthium L. }\end{array}$ & erba janca & {$[26,56,58,62,65,71]$} & Not Reported & -- & - & $\begin{array}{l}\text { Eupeptic, cholagogic, } \\
\text { antidyspeptic }\end{array}$ & Fh, Le & [27] \\
\hline $\begin{array}{c}\text { Centaurea } \\
\text { benedicta (L.) L. } \\
\end{array}$ & centarva & {$[56,62,65,68]$} & Not Reported & -- & - & Eupeptic & Fh, Le & {$[28]$} \\
\hline $\begin{array}{c}\text { Centaurea } \\
\text { centaurium L. }\end{array}$ & $\begin{array}{l}\text { centu } \\
\text { gruppa }\end{array}$ & [11] & Not Reported & -- & - & Antioxidant & Ro & [29] \\
\hline $\begin{array}{c}\text { Matricaria } \\
\text { chamomilla L. }\end{array}$ & gamumilla & [26] & $\begin{array}{c}\text { Digestive, sedative, } \\
\text { antispasmodic, antitussive }\end{array}$ & $\mathrm{Fh}$ & {$[49,50]$} & $\begin{array}{c}\text { Anti-inflammatory, } \\
\text { antibacterial, antifungal }\end{array}$ & $\mathrm{Fh}$ & [27] \\
\hline \multicolumn{9}{|l|}{ Boraginaceae } \\
\hline $\begin{array}{c}\text { Borago } \\
\text { officinalis } \mathrm{L} \text {. }\end{array}$ & erva pignola & {$[60]$} & $\begin{array}{c}\text { Diaphoretic, reddenings, } \\
\text { burns, sunburns, tussis, } \\
\text { rheumatisms, refreshing, } \\
\text { diuretic }\end{array}$ & Le, Ap & {$[49,50]$} & Anti-inflammatory & Oi & {$[27]$} \\
\hline $\begin{array}{l}\text { Heliotropium } \\
\text { europaeum L. }\end{array}$ & $\begin{array}{l}\text { Not } \\
\text { reported }\end{array}$ & {$[67]$} & Urinary inflammations & Ap & [49] & Antibacterial & Oi & {$[30]$} \\
\hline \multicolumn{9}{|l|}{ Capparaceae } \\
\hline $\begin{array}{l}\text { Capparis } \\
\text { spinosa L. }\end{array}$ & chiappara & {$[62]$} & Not Reported & -- & - & Antiviral & $\mathrm{Bl}$ & [31] \\
\hline \multicolumn{9}{|l|}{ Cactaceae } \\
\hline $\begin{array}{l}\text { Opuntia ficus } \\
\text { indica (L.) Mill.** }\end{array}$ & ficuniano & {$[60]$} & $\begin{array}{l}\text { Antispasmodic, } \\
\text { antidiarrhoeic, diuretic, to } \\
\text { treat bronchitis, }\end{array}$ & $\mathrm{Fl}, \mathrm{Fr}$ & [49] & Anti-inflammatory & Cld & {$[32]$} \\
\hline \multicolumn{9}{|l|}{ Cucurbitaceae } \\
\hline $\begin{array}{c}\text { Ecballium } \\
\text { elaterium }(\mathrm{L} .) \mathrm{A} . \\
\text { Rich. }^{* *} \\
\end{array}$ & cucummeru & [57] & Antirheumatic & $\mathrm{Fr}$ & [51] & Cholagogic & Frj & [33] \\
\hline \multicolumn{9}{|l|}{ Dryopteridaceae } \\
\hline $\begin{array}{l}\text { Dryopteris filix- } \\
\text { mas (L.) Schott. }\end{array}$ & $\begin{array}{c}\text { filici } \\
\text { masculu }\end{array}$ & {$[58]$} & $\begin{array}{c}\text { To treat sores provoked by } \\
\text { severe burns }\end{array}$ & Le & {$[49]$} & $\begin{array}{l}\text { Anti-helmintic, anti- } \\
\text { amebic, antiparasitic, } \\
\text { antiprotozoal }\end{array}$ & Ro & {$[34]$} \\
\hline
\end{tabular}


Table 2 Medical use of plants to heal malaria in Calabria, bio-pharmacological properties and current use in Calabrian folk medicine (Continued)

\begin{tabular}{|c|c|c|c|c|c|c|c|c|}
\hline \multicolumn{9}{|l|}{ Euphorbiaceae } \\
\hline $\begin{array}{c}\text { Ricinus } \\
\text { communis L. }\end{array}$ & ricinu & [26] & Not Reported & -— & -— & Laxative & Se & {$[28]$} \\
\hline \multicolumn{9}{|l|}{ Fabaceae } \\
\hline Lupinus albus L. & lupinu & {$[56,58,62,68]$} & To treat dermatitis in cattle & Ep & {$[50]$} & $\begin{array}{l}\text { Gingival anti- } \\
\text { inflammatory }\end{array}$ & $\mathrm{Se}$ & {$[35]$} \\
\hline \multicolumn{9}{|l|}{ Fagaceae } \\
\hline Quercus spp. & cerza & {$[55,58]$} & $\begin{array}{l}\text { Intestinal astringent, } \\
\text { detoxifying, cicatrizing }\end{array}$ & $\begin{array}{l}\text { Dried } \\
\text { bark of } \\
\text { young } \\
\text { stems } \\
\text { and } \\
\text { galls }\end{array}$ & {$[50]$} & $\begin{array}{l}\text { Anti-inflammatory, } \\
\text { antidiarrhoeic }\end{array}$ & $\mathrm{Ba}$ & {$[27]$} \\
\hline \multicolumn{9}{|l|}{ Gentianaceae } \\
\hline $\begin{array}{c}\text { Erythraea } \\
\text { centaurium (L.) } \\
\text { Borkh. } \\
\end{array}$ & brundulija & {$[11,60]$} & Not Reported & -— & -— & Eupeptic, antidyspeptic & Le, Fl & {$[27]$} \\
\hline $\begin{array}{c}\text { Gentiana lutea } \\
\mathrm{L} \text {. }\end{array}$ & genziana & {$[55]$} & Not Reported & -— & -— & Antidyspeptic, eupeptic & Ro & {$[27]$} \\
\hline \multicolumn{9}{|l|}{ Juglandaceae } \\
\hline Juglans regia L.* & nuci & [68] & $\begin{array}{l}\text { Vermifuge, anti-diarrhoeic, } \\
\text { stomachic, to remove } \\
\text { calluses, against excessive } \\
\text { feet perspiration }\end{array}$ & $\begin{array}{l}\mathrm{Hu}, \mathrm{Le}, \\
\mathrm{Fr}, \mathrm{Fle}\end{array}$ & [49-51] & Anti-inflammatory & Le & {$[27]$} \\
\hline \multicolumn{9}{|l|}{ Hyacinthaceae } \\
\hline $\begin{array}{c}\text { Urginea } \\
\text { maritima (L.) } \\
\text { Baker }\end{array}$ & cipuddazza & {$[56,62]$} & Not Reported & - - & - - & Cardiotonic & $\mathrm{Bl}$ & {$[28]$} \\
\hline \multicolumn{9}{|l|}{ Lamiaceae } \\
\hline $\begin{array}{l}\text { Ajuga } \\
\text { chamaepitys } \\
\text { Guss. }\end{array}$ & campezio & [72] & Not Reported & - & - & $\begin{array}{c}\text { anabolic, analgesic, anti- } \\
\text { arthritic, antibacterial, } \\
\text { antiestrogenic, } \\
\text { antifungal, anti- } \\
\text { inflammatory, anti- } \\
\text { hypertensive, } \\
\text { antileukemic, } \\
\text { antimalarial } \\
\text { antimycobacterial, } \\
\text { antioxidant, antipyretic, } \\
\text { cardiotonic, cytotoxic, } \\
\text { hypoglycemic, } \\
\text { vasorelaxing activity }\end{array}$ & Ep & {$[36]$} \\
\hline Ballota nigra L. & $\begin{array}{c}\text { marrobio } \\
\text { nero }\end{array}$ & [63] & Not Reported & - & - & Antioxidant & Le & {$[44]$} \\
\hline $\begin{array}{c}\text { Calamintha } \\
\text { nepeta (L.) Savi }\end{array}$ & nipitella & {$[64,69,70]$} & $\begin{array}{c}\text { To cure insect and snake } \\
\text { bites, cicatrizing }\end{array}$ & Fle, Fh & {$[49,50]$} & Antibacterial & Eoi & {$[45]$} \\
\hline $\begin{array}{l}\text { Hyssopus } \\
\text { officinalis } \mathrm{L} \text {. }\end{array}$ & issopu & [65] & Not Reported & -- & - - & Emmenagogue & Ro & {$[27]$} \\
\hline $\begin{array}{l}\text { Rosmarinus } \\
\text { officinalis L. }\end{array}$ & rosimarinu & {$[64,70,71]$} & $\begin{array}{l}\text { To ripen abscesses quickly, } \\
\text { carminative, digestive, to } \\
\text { speed up the recovery of } \\
\text { sores and wounds }\end{array}$ & $\begin{array}{c}\mathrm{Fl}, \mathrm{Le}, \\
\mathrm{Br}\end{array}$ & [49] & $\begin{array}{l}\text { Eupeptic, antidyspeptic, } \\
\text { emmenagogue, anti- } \\
\text { inflammatory }\end{array}$ & $\begin{array}{l}\text { Le, } \\
\text { Fh, } \\
\text { Eoi }\end{array}$ & {$[27]$} \\
\hline $\begin{array}{c}\text { Salvia officinalis } \\
\mathrm{L} \text {. }\end{array}$ & sarvia & {$[64,70,71]$} & $\begin{array}{l}\text { Digestive, antiasthmatic, to } \\
\text { speed up the recovery of } \\
\text { sores and wounds, to treat } \\
\text { tussis, to cure aphtas and } \\
\text { stomatitis, to treat swollen } \\
\text { testicles and related pains }\end{array}$ & $\begin{array}{l}\text { Le, Fl, } \\
\text { Dle }\end{array}$ & {$[49,50]$} & $\begin{array}{l}\text { Antioxidant, anti- } \\
\text { inflammatory }\end{array}$ & $\begin{array}{l}\text { Le, } \\
\text { Ap }\end{array}$ & {$[27]$} \\
\hline $\begin{array}{c}\text { Teucrium } \\
\text { chamaedrys L. }\end{array}$ & cametriu & {$[11,26,55,56,62,65]$} & Not Reported & -- & -- & Poisonous & - - & {$[37]$} \\
\hline
\end{tabular}


Table 2 Medical use of plants to heal malaria in Calabria, bio-pharmacological properties and current use in Calabrian folk medicine (Continued)

\begin{tabular}{|c|c|c|c|c|c|c|c|c|}
\hline Lythraceae & & & & & & & & \\
\hline $\begin{array}{l}\text { Punica } \\
\text { granatum L. }\end{array}$ & granato & {$[58]$} & Haemostatic, vermifuge & $\begin{array}{l}\text { Frb, } \\
\text { Rob }\end{array}$ & {$[51]$} & Emmenagogue & Rob & {$[27]$} \\
\hline \multicolumn{9}{|l|}{ Myrtaceae } \\
\hline Eucalyptus spp. & calipsi & {$[58,68]$} & $\begin{array}{l}\text { Antiseptic of the } \\
\text { respiratory tract }\end{array}$ & Le & {$[50]$} & $\begin{array}{c}\text { Antibacterial, anti- } \\
\text { inflammatory, } \\
\text { spasmolytic, expectorant }\end{array}$ & Le & {$[27]$} \\
\hline \multicolumn{9}{|l|}{ Oleaceae } \\
\hline $\begin{array}{c}\text { Olea europaea } \\
\text { L. }\end{array}$ & alivu & {$[62,68]$} & $\begin{array}{l}\text { Cholagogic, hypotensive, } \\
\text { astringent, suppurative, to } \\
\text { treat small burns, tooth } \\
\text { ache }\end{array}$ & $\begin{array}{l}\text { Le, Rfr, } \\
\text { Ba, Oi }\end{array}$ & {$[49-51]$} & $\begin{array}{l}\text { Hypotensive, diuretic, } \\
\text { spasmolytic, antipyretic }\end{array}$ & Le & {$[27]$} \\
\hline \multicolumn{9}{|l|}{ Papaveraceae } \\
\hline $\begin{array}{l}\text { Chelidonium } \\
\text { majus L. }\end{array}$ & cucumaju & {$[56]$} & $\begin{array}{c}\text { To treat warts, calluses, } \\
\text { gastric pains }\end{array}$ & La, Le & [49] & $\begin{array}{c}\text { Cholagogic, choleretic, } \\
\text { hypotensive, } \\
\text { antibacterial, antifungal, } \\
\text { antiviral, anti- } \\
\text { inflammatory, } \\
\text { antidyspeptic }\end{array}$ & $A p$ & {$[27]$} \\
\hline $\begin{array}{c}\text { Fumaria } \\
\text { officinalis L. }\end{array}$ & fumaria & {$[11]$} & Not Reported & - - & -— & Cholagogic, choleretic & $A p$ & {$[27]$} \\
\hline \multicolumn{9}{|l|}{ Piperaceae } \\
\hline Piper nigrum L. & pipi nivuro & {$[60,63,64]$} & Not Reported & -— & -— & $\begin{array}{l}\text { Antioxidant, anti- } \\
\text { inflammatory, anti- } \\
\text { diarrhoeal, eupeptic }\end{array}$ & $\mathrm{Se}$ & {$[46]$} \\
\hline \multicolumn{9}{|l|}{ Poaceae } \\
\hline Arundo donax L. & canna & {$[62]$} & $\begin{array}{c}\text { Haemostatic, cicatrising, to } \\
\text { treat throat inflammations } \\
\text { and bronchitis }\end{array}$ & $\mathrm{Sa}, \mathrm{Rh}$ & {$[49,50]$} & $\begin{array}{l}\text { Hypotensive, } \\
\text { spasmolytic }\end{array}$ & $\mathrm{Rh}$ & {$[38]$} \\
\hline $\begin{array}{l}\text { Cynodon } \\
\text { dactylon (L.) } \\
\text { Pers. }\end{array}$ & $\begin{array}{l}\text { acropastu, } \\
\text { addisa, } \\
\text { gramigna }\end{array}$ & {$[11,54]$} & $\begin{array}{l}\text { Diuretic, to alleviate } \\
\text { rheumatic pains, } \\
\text { inflammations of the } \\
\text { digestive and urogenital } \\
\text { system }\end{array}$ & $\begin{array}{l}\text { Ap, Rh, } \\
\text { Se }\end{array}$ & {$[49-51]$} & $\begin{array}{l}\text { Diuretic, anti- } \\
\text { inflammatory }\end{array}$ & Ro & {$[27]$} \\
\hline \multicolumn{9}{|l|}{ Rosaceae } \\
\hline $\begin{array}{c}\text { Prunus spinosa } \\
\text { L. }\end{array}$ & cucumele & {$[24,69,72,73]$} & Not Reported & - & -— & Anti-inflammatory & $\mathrm{Fr}$ & {$[27]$} \\
\hline \multicolumn{9}{|l|}{ Rutaceae } \\
\hline $\begin{array}{l}\text { Citrus bergamia } \\
\text { Risso }\end{array}$ & bergamotto & [64] & $\begin{array}{c}\text { Cicatrizing and antiseptic } \\
\text { for wounds and chilblains, } \\
\text { to cure anomalous vaginal } \\
\text { secretions, as a } \\
\text { contraceptive }\end{array}$ & Eoi & [49] & Antimicrobial & $\mathrm{Ba}$ & {$[47]$} \\
\hline $\begin{array}{l}\text { Citrus limonum } \\
\text { Risso }\end{array}$ & limuni & {$[11,56,65,74]$} & $\begin{array}{c}\text { Stomachic, to treat cough, } \\
\text { slimming agent, chilblains, } \\
\text { migraine (after } \\
\text { drunkenness), toothache, } \\
\text { rheumatisms, oral hollow } \\
\text { diseases }\end{array}$ & $\mathrm{Frj}, \mathrm{Fr}$ & [49] & Anti-inflammatory & Frj & [39] \\
\hline Ruta spp. & ruta & {$[64,70]$} & $\begin{array}{l}\text { Anti-helmintic, to treat } \\
\text { gastritis, abscesses, } \\
\text { rheumatic pains, headache, } \\
\text { intestinal inflammations } \\
\text { and eye reddening }\end{array}$ & Ap, Le & {$[49,50]$} & Antibacterial & Le & [48] \\
\hline \multicolumn{9}{|l|}{ Salicaceae } \\
\hline Salix spp. & salici & [58] & $\begin{array}{l}\text { Against fever and } \\
\text { rheumatic pains }\end{array}$ & $\mathrm{Ba}$ & [50] & $\begin{array}{c}\text { Antipyretic, anti- } \\
\text { inflammatory, analgesic }\end{array}$ & $\mathrm{Ba}$ & {$[27]$} \\
\hline
\end{tabular}


Table 2 Medical use of plants to heal malaria in Calabria, bio-pharmacological properties and current use in Calabrian folk medicine (Continued)

\begin{tabular}{|c|c|c|c|c|c|c|c|c|}
\hline Smilacaceae & & & & & & & & \\
\hline Smilax aspera L. & $\begin{array}{l}\text { strazza } \\
\text { buttuni }\end{array}$ & [11] & Not Reported & -— & -— & Adaptogen & Ro & [27] \\
\hline \multicolumn{9}{|l|}{ Solanaceae } \\
\hline $\begin{array}{c}\text { Solanum } \\
\text { dulcamara } \mathrm{L} .\end{array}$ & durcamara & [11] & Not Reported & - & - & To treat dermatitis & St & {$[27]$} \\
\hline $\begin{array}{l}\text { Capsicum } \\
\text { annuum L. }\end{array}$ & pipariaddru & {$[56,60,62,75]$} & $\begin{array}{c}\text { To rise blood flow to } \\
\text { superficial tissues }\end{array}$ & $\mathrm{Fr}$ & {$[49]$} & $\begin{array}{c}\text { Antidyspeptic, anti- } \\
\text { inflammatory }\end{array}$ & $\mathrm{Fr}$ & {$[27]$} \\
\hline $\begin{array}{l}\text { Capsicum } \\
\text { annuum L. Var. } \\
\text { acuminatum } \\
\text { Fing. }\end{array}$ & pipi & {$[55,57,60,68,76]$} & Revulsive & $\mathrm{Fr}$ & [49] & Antioxidant & $\mathrm{Fr}$ & {$[40]$} \\
\hline \multicolumn{9}{|l|}{ Tamaricaceae } \\
\hline Tamarix spp. & vruca & {$[24,65]$} & Not Reported & -— & -— & Antioxidant, antibacterial & $\mathrm{Fl}$, Le & [41] \\
\hline \multicolumn{9}{|l|}{ Urticaceae } \\
\hline $\begin{array}{l}\text { Parietaria } \\
\text { officinalis } \mathrm{L} \text {. }\end{array}$ & erba 'i muru & {$[56,62]$} & $\begin{array}{c}\text { Diuretic, depurative, } \\
\text { cholagogue, to treat } \\
\text { bruises, haematoma, } \\
\text { kidney stones, abscesses, } \\
\text { skin inflammations, viper } \\
\text { bite }\end{array}$ & $\begin{array}{l}\text { Le, Ro, } \\
\text { Ap, Ep }\end{array}$ & {$[49-51]$} & Diuretic, uricosuric & Ap & [42] \\
\hline Urtica dioica $\mathrm{L}$. & urdica & [62] & $\begin{array}{c}\text { Antirheumatic, } \\
\text { hepatoprotective, to treat } \\
\text { haemorrhoids, renal } \\
\text { troubles }\end{array}$ & $\begin{array}{l}\text { Ap, Ro, } \\
\text { Le, To }\end{array}$ & {$[49,50]$} & $\begin{array}{l}\text { Diuretic, anti- } \\
\text { inflammatory }\end{array}$ & $\begin{array}{l}\text { Ap, } \\
\text { Ro }\end{array}$ & [27] \\
\hline Urtica urens L. & $\begin{array}{l}\text { urdica } \\
\text { piccola }\end{array}$ & [63] & Not Reported & -- & - & $\begin{array}{l}\text { Diuretic, anti- } \\
\text { inflammatory }\end{array}$ & $\begin{array}{l}\text { Ap, } \\
\text { Ro }\end{array}$ & [27] \\
\hline \multicolumn{9}{|l|}{ Valerianaceae } \\
\hline $\begin{array}{l}\text { Valeriana } \\
\text { officinalis L. }\end{array}$ & malariana & [65] & Not Reported & - & - & Sedative & Ro & {$[27]$} \\
\hline \multicolumn{9}{|l|}{ Verbenaceae } \\
\hline $\begin{array}{c}\text { Verbena } \\
\text { officinalis } L . *\end{array}$ & $\begin{array}{l}\text { erba di la } \\
\text { crucivia }\end{array}$ & {$[56,57,62]$} & Not Reported & - & - & $\begin{array}{c}\text { Anti-inflammatory, } \\
\text { analgesic }\end{array}$ & Le & [43] \\
\hline
\end{tabular}

* Plant used like magic remedy too (see text); ** plants used like magic remedy only (see text); Used parts of plant: Ap aerial part; Ba bark; BI bulb; Br branches; Bu bud; $\mathrm{Cl}$ cloves; Cld cladodes; Dle dry leaves; Dfh dry flower heads; Eoi essential oil; Ep entire plant; Fh flower heads; Fi feminine inflorescences; Fl flowers; Fle fresh leaves; Fr fruit; Frb fruit bark; Frj fruit juice; Hu husk; La latex; Le leaves; Oi oil; Rfr ripe fruit; Rh rhizome; Ro root; Rob root bark; Sa sap; Se seeds; St stem; To tops;

${ }^{\circ}$ Current use drawn from recent fieldworks

○ The properties relating to certain species of the genus Ajuga

dung [55]. As three "Cantarelle" (Cantharis vescicatoria) minced in water were used as a diuretic against dropsy [56].

Calabrian people believed without any doubt in the remedies described above; however, they also used numerous plants to cure malaria. Some of these plants are still currently used in Calabrian folk medicine to cure various diseases [Table 1]. Many decoctions or infusions of various herbaceous species were used to cure malariaassociated fevers. These herbaceous plants included "issopu" (Hyssopus officinalis L.), "valariana" (Valeriana officinalis L.), "filici masculu" (Dryopteris filix-mas (L.) Schott.), "lupinu" (Lupinus albus L.), "cametriu" (Teucrium chamaedrys L.), "brundulija" (Erythraea centaurium (L.) Borkh.), "centu gruppa" (Centaurea centaurium
L.), "centarva" (Centaurea benedicta L.), "sarvia" (Salvia officinalis L.), "rosamarinu" (Rosmarinus officunalis L.), "nepitella" (Calamintha nepeta (L.) Savi), "ruta" (Ruta spp.), "erva janca" (Artemisia absinthium L.), "cipuddra" (Allium cepa L.), "agliu" (Allium sativum L.), "millefoglio" (Achillea millefolium L.), "erva pignola" (Borago officinalis L.), "marrobio nero" (Ballota nigra L.), "campezio" (Ajuga chamaepitys Guss.), "elitropia" (Heliotropium europaeum L.) (which was ingested with white wine), "genziana" (Gentiana lutea L.) and "erba i la crucivia" (Verbena officinalis L.) [11,26,55-58,60,62-65,67-72]. Some ligneous species must be added to this list, particularly the following plants: the aerial parts of "vruca" (Tamarix spp.), and "leandru" (Nerium oleander L.); the leaves of "alivu" (Olea europaea L.) and "calipsi" 
Table 3 Medical use of plants to heal malaria in Calabria, mentioned by historical sources used for the research

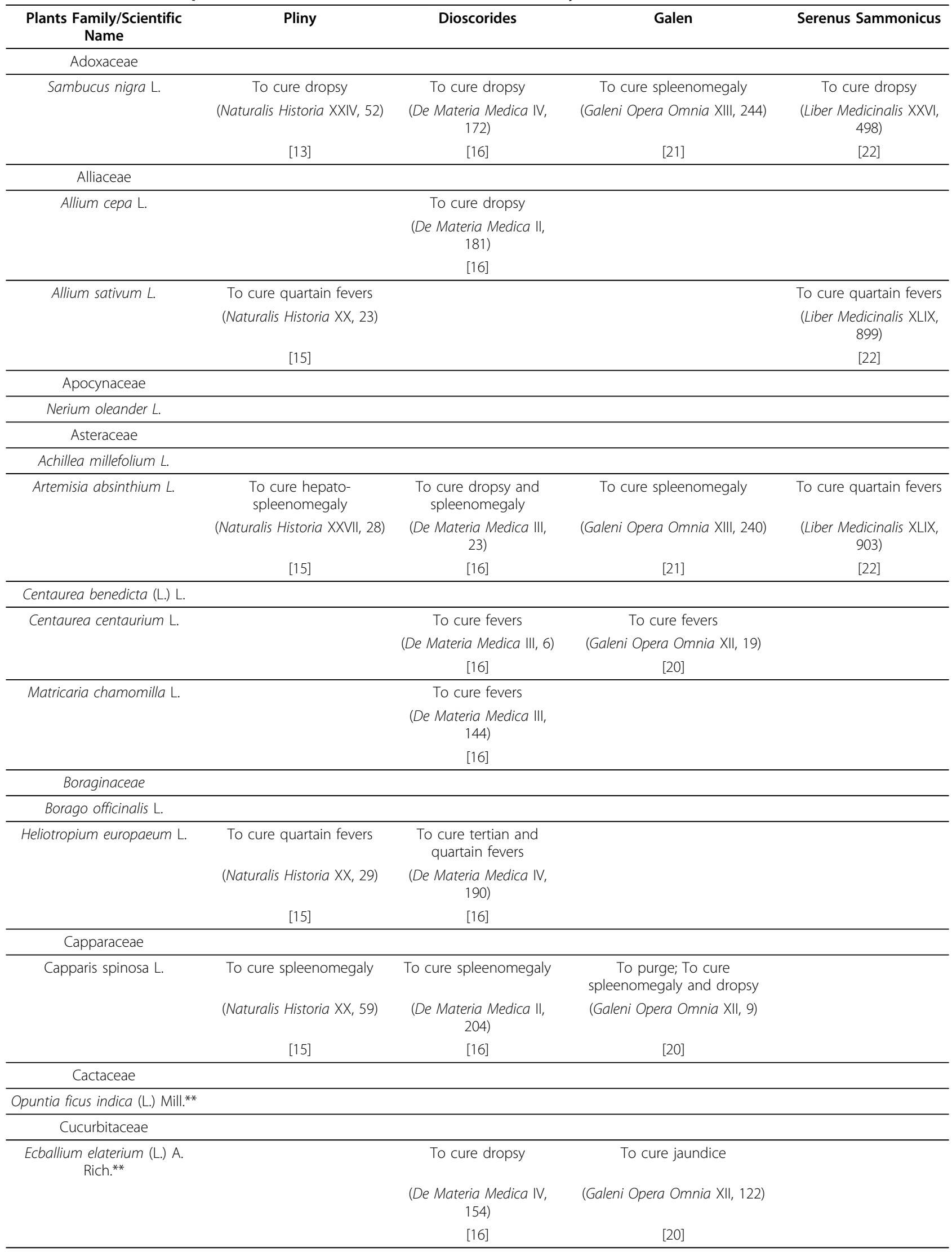


Table 3 Medical use of plants to heal malaria in Calabria, mentioned by historical sources used for the research (Continued)

\begin{tabular}{|c|c|c|c|c|}
\hline Dryopteridaceae & & & & \\
\hline \multirow[t]{3}{*}{ Dryopteris filix-mas (L.) Schott. } & & To cure spleenomegaly & & To cure dropsy \\
\hline & & $\begin{array}{c}\text { (De Materia Medica IV, } \\
\text { 158) }\end{array}$ & & $\begin{array}{l}\text { (Liber Medicinalis XVI, } \\
\text { 511) }\end{array}$ \\
\hline & & {$[16]$} & & [22] \\
\hline \multicolumn{5}{|l|}{ Euphorbiaceae } \\
\hline \multirow[t]{3}{*}{ Ricinus communis $\mathrm{L}$. } & & $\begin{array}{c}\text { To purge; To cure } \\
\text { dropsy }\end{array}$ & To purge & \\
\hline & & $\begin{array}{c}\text { (De Materia Medica IV, } \\
141)\end{array}$ & (Galeni Opera Omnia XII, 26) & \\
\hline & & [16] & [20] & \\
\hline \multicolumn{5}{|l|}{ Fabaceae } \\
\hline \multirow[t]{3}{*}{ Lupinus albus L. } & To cure spleenomegaly & To cure spleenomegaly & & \\
\hline & (Naturalis Historia XXII, 74) & $\begin{array}{c}\text { (De Materia Medica II, } \\
\text { 132) }\end{array}$ & & \\
\hline & {$[15]$} & {$[16]$} & & \\
\hline \multicolumn{5}{|l|}{ Fagaceae } \\
\hline \multirow[t]{3}{*}{ Quercus spp. } & & To expel urine & & \\
\hline & & $\begin{array}{l}\text { (De Materia Medica I, } \\
143 \text { ) }\end{array}$ & & \\
\hline & & [16] & & \\
\hline \multicolumn{5}{|l|}{ Gentianaceae } \\
\hline \multirow[t]{3}{*}{ Erythraea centaurium (L.) Borkh. } & & To cure dropsy & To cure spleenomegaly & \\
\hline & & (De Materia Medica III, 7) & (Galeni Opera Omnia XII, 20) & \\
\hline & & {$[16]$} & [20] & \\
\hline \multirow[t]{3}{*}{ Gentiana lutea L. } & & To cure hepatomegaly & & \\
\hline & & (De Materia Medica III, 3) & & \\
\hline & & {$[16]$} & & \\
\hline \multicolumn{5}{|l|}{ Juglandaceae } \\
\hline \multicolumn{5}{|l|}{ Juglans regia $\mathrm{L} . *$} \\
\hline \multicolumn{5}{|l|}{ Hyacinthaceae } \\
\hline \multirow[t]{3}{*}{ Urginea maritima (L.) Baker } & To cure dropsy & $\begin{array}{l}\text { To cure dropsy and } \\
\text { jaundice }\end{array}$ & $\begin{array}{l}\text { To cure hepato-spleenomegaly; } \\
\text { To expel urine }\end{array}$ & \\
\hline & (Naturalis Historia XX, 100) & $\begin{array}{c}\text { (De Materia Medica II, } \\
\text { 102) }\end{array}$ & $\begin{array}{c}\text { (Galeni Opera Omnia XI, 746, } \\
\text { 749) }\end{array}$ & \\
\hline & [15] & [16] & [18] & \\
\hline \multicolumn{5}{|l|}{ Lamiaceae } \\
\hline \multirow[t]{3}{*}{ Ajuga chamaepitys Guss. } & To cure dropsy & To cure jaundice & $\begin{array}{l}\text { To expel urine; To cure } \\
\text { spleenomegaly }\end{array}$ & \\
\hline & (Naturalis Historia XXIV, 30) & $\begin{array}{c}\text { (De Materia Medica III, } \\
175)\end{array}$ & $\begin{array}{c}\text { (Galeni Opera Omnia XII 155; XIII, } \\
240)\end{array}$ & \\
\hline & [13] & [16] & {$[20,21]$} & \\
\hline \multirow[t]{3}{*}{ Ballota nigra $\mathrm{L}$. } & & & To cure spleenomegaly & $\begin{array}{l}\text { To cure hepato- } \\
\text { splenomegaly }\end{array}$ \\
\hline & & & (Galeni Opera Omnia XII, 108) & $\begin{array}{l}\text { (Liber Medicinalis XXII, } \\
417 \text { ) }\end{array}$ \\
\hline & & & [20] & {$[22]$} \\
\hline \multirow[t]{3}{*}{ Calamintha nepeta (L.) Savi } & & To cure jaundice & To cure dropsy & $\begin{array}{l}\text { To cure spleenomegaly } \\
\text { and dropsy }\end{array}$ \\
\hline & & $\begin{array}{l}\text { (De Materia Medica III, } \\
\text { 28) }\end{array}$ & (Galeni Opera Omnia XIII, 264) & $\begin{array}{l}\text { (Liber Medicinalis XXII, } \\
\quad 419 ; \text { XVI, 504) }\end{array}$ \\
\hline & & [16] & [21] & {$[22]$} \\
\hline
\end{tabular}


Table 3 Medical use of plants to heal malaria in Calabria, mentioned by historical sources used for the research (Continued)

\begin{tabular}{|c|c|c|c|c|}
\hline \multirow[t]{2}{*}{ Hyssopus officinalis $L$. } & $\begin{array}{l}\text { To cure spleenomegaly } \\
\text { (Naturalis Historia XXVI, 48) }\end{array}$ & $\begin{array}{l}\text { To cure dropsy and } \\
\text { spleenomegaly } \\
\text { (De Materia Medica III, } \\
\text { 28) }\end{array}$ & $\begin{array}{c}\text { To cure dropsy } \\
\text { (Galeni Opera Omnia XIII, 263) }\end{array}$ & \\
\hline & [13] & [16] & [21] & \\
\hline \multirow[t]{3}{*}{ Rosmarinus officinalis $\mathrm{L}$. } & $\begin{array}{l}\text { To cure hepato- } \\
\text { splenomegaly }\end{array}$ & To cure jaundice & To cure jaundice & $\begin{array}{l}\text { To cure hepato- } \\
\text { splenomegaly }\end{array}$ \\
\hline & (Naturalis Historia XXIV, 59) & $\begin{array}{c}\text { (De Materia Medica III, } \\
\text { 89) }\end{array}$ & (Galeni Opera Omnia XII, 60) & $\begin{array}{c}\text { (Liber Medicinalis XXII, } \\
408)\end{array}$ \\
\hline & [13] & {$[16]$} & {$[20]$} & [22] \\
\hline \multirow[t]{3}{*}{ Salvia officinalis $\mathrm{L}$. } & & To expel urine & & To cure hepatomegaly \\
\hline & & $\begin{array}{c}\text { (De Materia Medica III, } \\
35)\end{array}$ & & $\begin{array}{c}\text { (Liber Medicinalis XXI, } \\
\text { 381) }\end{array}$ \\
\hline & & {$[16]$} & & [22] \\
\hline \multirow[t]{3}{*}{ Teucrium chamaedrys L. } & $\begin{array}{c}\text { To cure spleenomegaly } \\
\text { and dropsy }\end{array}$ & To cure spleenomegaly & To cure spleen; To expel urine & \\
\hline & $\begin{array}{c}\text { (Naturalis Historia XXIV, } \\
131 \text { ) }\end{array}$ & $\begin{array}{c}\text { (De Materia Medica III, } \\
\text { 102) }\end{array}$ & (Galeni Opera Omnia XII, 153) & \\
\hline & [13] & [16] & {$[20]$} & \\
\hline \multicolumn{5}{|l|}{ Lythraceae } \\
\hline \multicolumn{5}{|l|}{ Punica granatum $\mathrm{L}$. } \\
\hline \multicolumn{5}{|l|}{ Myrtaceae } \\
\hline \multicolumn{5}{|l|}{ Eucalyptus spp. } \\
\hline \multicolumn{5}{|l|}{ Oleaceae } \\
\hline \multirow[t]{3}{*}{ Olea europaea L. } & & To expel urine & & \\
\hline & & $\begin{array}{c}\text { (De Materia Medica I, } \\
141 \text { ) }\end{array}$ & & \\
\hline & & {$[16]$} & & \\
\hline \multicolumn{5}{|l|}{ Papaveraceae } \\
\hline \multirow[t]{3}{*}{ Chelidonium majus L. } & & To cure jaundice & To cure fevers & \\
\hline & & $\begin{array}{c}\text { (De Materia Medica II, } \\
211 \text { ) }\end{array}$ & (Galeni Opera Omnia XII, 156) & \\
\hline & & {$[16]$} & [20] & \\
\hline \multirow[t]{3}{*}{ Fumaria officinalis $\mathrm{L}$. } & & To expel urine & To expel urine & \\
\hline & & $\begin{array}{c}\text { (De Materia Medica IV, } \\
108)\end{array}$ & (Galeni Opera Omnia XII, 8) & \\
\hline & & [16] & [20] & \\
\hline \multicolumn{5}{|l|}{ Piperaceae } \\
\hline \multirow[t]{2}{*}{ Piper nigrum L. } & & $\begin{array}{c}\text { To cure periodical fevers } \\
\text { (De Materia Medica II, } \\
\text { 158) }\end{array}$ & $\begin{array}{c}\text { To cure quartain fevers } \\
\text { (Galeni Opera Omnia XIV, 524) }\end{array}$ & $\begin{array}{c}\text { To cure hepatomegaly } \\
\text { (Liber Medicinalis XXI, } \\
\text { 384) }\end{array}$ \\
\hline & & [16] & [19] & [22] \\
\hline \multicolumn{5}{|l|}{ Poaceae } \\
\hline \multirow[t]{3}{*}{ Arundo donax L. } & To cure dropsy & & & \\
\hline & (Naturalis Historia XXIV, 50) & & & \\
\hline & [13] & & & \\
\hline \multirow[t]{3}{*}{ Cynodon dactylon (L.) Pers. } & & & To expel urine & \\
\hline & & & (Galeni Opera Omnia XI, 810) & \\
\hline & & & [18] & \\
\hline \multicolumn{5}{|l|}{ Rosaceae } \\
\hline \multicolumn{5}{|l|}{ Prunus spinosa $\mathrm{L}$. } \\
\hline \multicolumn{5}{|l|}{ Rutaceae } \\
\hline Citrus bergamia Risso & & & & \\
\hline
\end{tabular}


Table 3 Medical use of plants to heal malaria in Calabria, mentioned by historical sources used for the research (Continued)

\begin{tabular}{|c|c|c|c|c|}
\hline Citrus limonum Risso & & & & \\
\hline \multirow[t]{3}{*}{ Ruta spp. } & & To cure dropsy & To expel urine; to cure dropsy & \\
\hline & & $\begin{array}{l}\text { (De Materia Medica III, } \\
45 \text { ) }\end{array}$ & $\begin{array}{c}\text { (Galeni Opera Omnia XII, 101; XIII, } \\
\text { 257) }\end{array}$ & \\
\hline & & [16] & {$[20,21]$} & \\
\hline \multicolumn{5}{|l|}{ Salicaceae } \\
\hline \multicolumn{5}{|l|}{ Salix spp. } \\
\hline \multicolumn{5}{|l|}{ Smilacaceae } \\
\hline \multicolumn{5}{|l|}{ Smilax aspera $\mathrm{L}$. } \\
\hline \multicolumn{5}{|l|}{ Solanaceae } \\
\hline \multirow[t]{3}{*}{ Solanum dulcamara $\mathrm{L}$. } & & & To expel urine & \\
\hline & & & (Galeni Opera Omnia XII, 145) & \\
\hline & & & {$[20]$} & \\
\hline \multicolumn{5}{|l|}{ Capsicum annuum $\mathrm{L}$. } \\
\hline \multicolumn{5}{|l|}{$\begin{array}{l}\text { Capsicum annuum L. Var. } \\
\text { acuminatum Fing. }\end{array}$} \\
\hline \multicolumn{5}{|l|}{ Tamaricaceae } \\
\hline \multirow[t]{2}{*}{ Tamarix spp. } & $\begin{array}{c}\text { To cure spleenomegaly } \\
\text { (Naturalis Historia XXIV, 61) }\end{array}$ & $\begin{array}{c}\text { To cure spleenomegaly } \\
\text { (De Materia Medica I, } \\
116)\end{array}$ & $\begin{array}{l}\text { To cure spleenomegaly } \\
\text { (Galeni Opera Omnia XII, 80) }\end{array}$ & $\begin{array}{c}\text { To cure spleenomegaly } \\
\text { (Liber Medicinalis XXII, } \\
\text { 408) }\end{array}$ \\
\hline & [15] & [16] & [20] & [22] \\
\hline \multicolumn{5}{|l|}{ Urticaceae } \\
\hline \multicolumn{5}{|l|}{ Parietaria officinalis $\mathrm{L}$. } \\
\hline \multirow[t]{3}{*}{ Urtica dioica $\mathrm{L}$., Urtica urens $\mathrm{L}$. } & $\begin{array}{l}\text { To cure spleenomegaly; to } \\
\text { expel urine }\end{array}$ & To cure spleenomegaly & & \\
\hline & (Naturalis Historia XXII, 15) & $\begin{array}{c}\text { (De Materia Medica IV, } \\
102)\end{array}$ & & \\
\hline & [15] & {$[16]$} & & \\
\hline \multicolumn{5}{|l|}{ Valerianaceae } \\
\hline \multirow[t]{3}{*}{ Valeriana officinalis $L$. } & & To cure jaundice & To expel urine & \\
\hline & & (De Materia Medica I, 6) & (Galeni Opera Omnia XII, 85) & \\
\hline & & {$[16]$} & {$[20]$} & \\
\hline \multicolumn{5}{|l|}{ Verbenaceae } \\
\hline \multirow[t]{3}{*}{ Verbena officinalis $\mathrm{L}$. } & To cure fevers & $\begin{array}{l}\text { To cure tertian and } \\
\text { quartain fevers }\end{array}$ & & \\
\hline & (Naturalis Historia XXV, 59) & $\begin{array}{l}\text { (De Materia Medica IV, } \\
61 \text { ) }\end{array}$ & & \\
\hline & [13] & [16] & & \\
\hline
\end{tabular}

(Eucalyptus spp.); the roots of "granato" (Punica granatum L.), "cucumele" (Prunus spinosa L.), "savucu" (Sambucus nigra L.), and "cerza” (Quercus spp.). The bark of "cucumele", "cerza" and "salici" (Salix spp.) was also used [24,26,55,58,62,65,68,69,72,73].

Other remedies were similarly efficacious; these included the mesocarp of "nuci" (Juglans regia L.) chopped finely and mixed with wine, "bergamotto" (Citrus bergamia Risso), "limuni" (Citrus limonum Risso, which was broken, boiled and maintained fresh overnight, then drunk at breakfast for three mornings), "pipi nivuru" (Piper nigrum
L.), "pepe arsente” (Capsicum annuum L.) and ten bitter seeds of decorticated "lupinu" (Lupinus albus L.), taken in the morning [11,56,58,60,62-65,68,74,75].

Other than fever, the most evident symptoms of malaria are hepato-spleenomegaly and dropsy. We also found descriptions of several remedies for these symptoms. There were many cures for hepato-spleenomegaly: a decoction of the root of "chiappara" (Capparis spinosa L.), or of "acropistu" (Urtica dioica L., Urtica urens L.), the latter taken together with potassium nitrate in the morning; eating "cipuddra" (Allium cepa L.) or "pipi" 
Table 4 Medical use of animals to heal malaria in Calabria, mentioned by historical sources used for the research

\begin{tabular}{|c|c|c|c|c|}
\hline Animals & Pliny & Dioscorides & Galen & Serenus Sammonicus \\
\hline \multirow[t]{3}{*}{ Cimex lecturalius } & To cure quartain fevers & To cure quartain fevers & & To cure tertian fevers \\
\hline & (Naturalis Historia XXIX, 17) & (De Materia Medica II, 36) & & (Liber Medicinalis XLIX, 921) \\
\hline & [14] & [16] & & [22] \\
\hline \multirow[t]{3}{*}{ Homo sapiens } & & To cure dropsy & & \\
\hline & & (De Materia Medica II, 99) & & \\
\hline & & [16] & & \\
\hline \multirow[t]{3}{*}{ Lumbricus terrestris } & & To cure tertian fevers & & \\
\hline & & (De Materia Medica II, 99) & & \\
\hline & & [16] & & \\
\hline \multirow[t]{3}{*}{ Spider } & To cure quartain fevers & To cure quartain fevers & & \\
\hline & (Naturalis Historia XXX, 30) & (De Materia Medica II, 48) & & \\
\hline & [14] & [16] & & \\
\hline \multirow[t]{3}{*}{ Vipera aspis } & To cure fevers & & & \\
\hline & (Naturalis Historia XXX, 30) & & & \\
\hline & [14] & & & \\
\hline \multirow[t]{3}{*}{ Capra hircus } & & To cure hepatomegaly & To cure spleenomegaly and dropsy & \\
\hline & & (De Materia Medica II, 98) & (Galeni Opera Omnia XII, 297; XIII, 263) & \\
\hline & & [16] & {$[20,21]$} & \\
\hline \multirow[t]{3}{*}{ Erinaceus europaeus } & To cure dropsy & To cure dropsy & & \\
\hline & (Naturalis Historia XXX, 30) & (De Materia Medica II, 2) & & \\
\hline & {$[14]$} & {$[16]$} & & \\
\hline \multirow[t]{3}{*}{ Cantharis vescicatoria } & To cure dropsy & & To expel urine & \\
\hline & (Naturalis Historia XXIX, 96) & & (Galeni Opera Omnia XII, 363) & \\
\hline & [14] & & [20] & \\
\hline \multirow[t]{3}{*}{ Snake } & To cure quartain fevers & & & \\
\hline & (Naturalis Historia XXX, 30) & & & \\
\hline & [14] & & & \\
\hline \multirow[t]{3}{*}{ Bos taurus } & & & To cure dropsy & \\
\hline & & & (Galeni Opera Omnia XIII, 263) & \\
\hline & & & [21] & \\
\hline
\end{tabular}

(Capsicum annuum L. Var. acuminatum Fing.), the latter together with a strong wine. Finally, another remedy involved the use of "cucumaju" (Chelidonium majus L.) [55-57,60,63,68,76].

To treat dropsy, which was called "acqua "ntà panza" (water in the stomach), Calabrian people used several diuretic remedies, such as "erba i muru" (Parietaria officinalis L.), "cipuddazza" (Urginea maritima (L.) Baker), and "cametriu" (Teucrium chamaedrys L.) $[11,26,55,56,62,66]$.

\section{Magic remedies}

Because of the presence of malaria in the daily lives of Calabrian people, this disease was considered a normal life trouble; however, its most dangerous and deadly forms were considered by Calabrian people as a condition of supernatural nature. Therefore, they resorted to magic remedies that were believed to "link" the disease. These included, in particular, wearing a "nuci trischéra o a tri guarri" (a three-valve walnut shell) (Juglans regia L.), a spider that was enclosed between two shells of a walnut or skin, skeleton and fangs of snake, the latter extracted when animal was still alive, as it was believed that the disease would then affect the walnut, the spider or the parts of the snake, and not the subjects who wore these amulets [65,77]. Furthermore, a live "carpurita" (Pachyiulus communis) was sewn into the clothes of the affected subject (without the patient realizing it) or a "paletta" (Opuntia ficus-indica (L.) Mill.) was placed near the fireplace. It was believed that when the animal died, or when the stem of the plant dried, the fever or the hepato-spleenomegaly would disappear $[26,60]$. In an analogy with the ancient belief in the therapeutic principle of "contact", to defeat spleenomegaly Calabrians were encouraged to place "erba i la crucivia" (Verbena officinalis L.) on the abdomen of the affected subject before sleeping, as it would absorb the "bad 
blood" $[56,57,62]$. Finally, every morning the affected subject had to urinate on "cucuzzielli acriesti maturi", the fruits of Ecballium elaterium (L.) A. Rich., to transfer the disease from the subject to the fruit [57].

\section{Religious remedies}

Calabrian people alternated or combined both empirical and magical remedies and, very often, used prayers and acts of devotion, as diseases were believed to be associated to divine punishment. Thus, in Cosenza (Northern Calabria) the "Madonna della Febbre" was invoked with prayers, ex voto and pilgrimages [78]; in Castrovillari (province of Cosenza), the prayer to the "Madonna d'Itria" was as follows: "Madonna mia 'i L'Itria, chi stai 'nganna a'sta jumara fammi passà 'sta freva 'i quartana c'u jurnu tuju non vugghiu mangià panu" ("My Lady of Itria, close to the river, let the fever out and on your commemoration day I will not eat bread") [79].

\section{Discussion}

The methodology based on the analysis of historical sources regarding Calabrian folk medicine remedies for the prophylaxis and treatment of malaria, if not compared with similar studies, can be considered a case study where the ordinary methodologies of ethno-medical-biological research are combined with the methodologies pertaining to historical-anthropological sciences. In addition, this is part of a debate regarding the association between ethnobotany and ethnopharmacology and other disciplines, to improve our understanding of the human usage of plants [80]. Moreover, this work complies with De Natale et al. [81], who created a database of the historical use of plants in the popular medicine of the Mediterranean basin. However, this study has revealed some interesting and heterogeneous features regarding Calabrian popular medicine practices used to prevent and treat malaria, some of which were inherited by the Calabrian people from the "expert medicine" of the past centuries.

The first type of practices that we have described were characterized by a rational approach. Indeed, the use of medicinal plants, $69 \%$ of which $(36 / 52)$ is recognized by the current pharmacopoeia as having some pharmacological/biological properties, succeeded in assuaging temporarily the most evident sufferings associated with the disease (fever, hepato-spleenomegaly, asthenia and dropsy) as well as its complications, such as the proneness to bacterial infections, even if did not cure the malarial infection. 23\% (12/52) of the plants which were used by Calabrian people to treat malaria, have pharmacological/biological properties which did not allow to relieve the symptoms of malaria; however, they did not damage the affected subjects. Finally, $8 \%(4 / 52)$ of these plants were characterized by some pharmacological/ biological properties which could be harmful for a malarial subject; or these properties could even be poisonous both for the malarial and the healthy subject.

The second type of practices were linked to the magic tradition of Calabrian folk medicine which, like the traditions of all Southern Italian regions, is rich in myths, symbolism and fantastic representations [82]. Thus, malaria became a synonym of "malia", or, as Pasquarelli [83] affirmed, it became "an aspect of paludism". Malaria was thought to be a consequence of a malefic element that affected the behaviour and the life of an individual; therefore, only a magic cure could remedy the disease.

The third type of practices were characterized by a strong principle of ineluctability, which is currently present among the Calabrian society: the sick entrusted God with prayers or acts of devotion, with the conviction that only God would be able to provide recovery from the disease.

\section{Conclusions}

The use of plants combined with other cures, such as the use of spiders, cantharis and leeches, represents prophylactic or therapeutic elements inherited from ancient medical science, some of which were still used to treat malaria in hospitals and in general by $19^{\text {th }}$-century physicians, before the introduction of quinine. This element is very interesting; while the empirical and magic remedies were not based on the symptomatology of the disease (they were rather "psychological and protective" elements [82]), the use of plants represented a real treatment, and served as a popular medicine base to treat various diseases.

In conclusion, the remedies described in this work allow us to establish the link between malaria and Calabrian people, so that Turner's statement that "the more widely or intensively a plant is used, the greater is its cultural significance" [84] can, in this case, be extended to malaria; the more folk remedies are used to cure malaria, the greater is the significance of its historical, medical and social meaning.

\section{Authors' contributions}

GT conceived of the study, collected and analyzed the data, drafted the manuscript. AT and AP supervised the work at all its stages.

\section{Competing interests}

The authors declare that they have no competing interests.

Received: 18 September 2009 Accepted: 18 September 2010 Published: 18 September 2010

\section{References}

1. Capasso L: The origin of human malaria. International Journal of Anthropology 1998, 13:165-175.

2. Givigliano GP: Land and Malaria in the Brutii. International Journal of Anthropology 1998, 3-4:181-194. 
3. Tognotti E: La malaria in Sardegna. Per una storia del paludismo nel Mezzogiorno (1880-1950) Milano: Franco Angeli Editore 1996.

4. Coluzzi A: L'eradicazione della malaria. Una sfida al mondo. Annali della sanità pubblica 1961, 22:241-253.

5. Tagarelli A: Distribuzione geografica della malaria. La Malaria in Calabria Comitato per gli studi storico e scientifici della malaria in Calabria. Castellammare di Stabia: Grafiche Somma 1997, 117-119.

6. Genovese F: Terremoti, bradisismi ed altri fenomeni geologici in rapporto alla malaria locale. La Malariologia 1921, VIl:13-19.

7. Misefori E: Storia sociale della Calabria Milano: Jaca Book 1974

8. Capra A, Consoli S, Russo A, Scicolone B: Variabilità climatica in Calabria ed effetti su alcune grandezze agrometereologiche. In IX Convegno Nazionale dell'Associazione Italiana di Ingegneria Agraria: 12-16 settembre 2009; Ischia (NA) Edited by: Doppia Voce 2009, 2-20.

9. Placanica A: Storia della Calabria: dall'antichità ai giorni nostri Roma: Donzelli Editore 1999.

10. Altimari F, Savoia LM: I dialetti italo-albanesi. Studi linguistici e storico-culturali sulle comunità arbëreshe Roma: Bulzoni Editore 1994

11. Lombardi Satriani R: Credenze popolari calabresi Napoli: Edizioni De Simone 1951

12. Cavalcanti O: La cultura subalterna in Calabria: profilo storico degli studi e bibliografia Roma, Reggio Calabria: Casa del libro 1982.

13. Jones WHS: Pliny, Natural History Cambridge (Massachusets): Harvard University Press and London: William Heinemann LTD 1966, VII:XXIV-XXVII.

14. Jones WHS: Pliny, Natural History Cambridge (Massachusets): Harvard University Press and London: William Heinemann LTD 1963, VIII:XXVIII-XXXII.

15. Jones WHS: Pliny, Natural History Cambridge (Massachusets): Harvard University Press and London: William Heinemann LTD 1969, VI:XX-XXIII.

16. Kühn CG: Pedanii Dioscoridis Anazarbei De Materia Medica Tomus I Lipsiae: Officina Libraria Car. Knoblochii 1829.

17. Kühn CG: Claudii Galeni Opera Omnia Tomus X Lipsiae: Officina Libraria Car. Knoblochii 1821

18. Kühn CG: Claudii Galeni Opera Omnia Tomus XI Lipsiae: Officina Libraria Car. Knoblochii 1826

19. Kühn CG: Claudii Galeni Opera Omnia Tomus XIV Lipsiae: Officina Libraria Car. Knoblochii 1827.

20. Kühn CG: Claudii Galeni Opera Omnia Tomus XII Lipsiae: Officina Libraria Car. Knoblochii 1826

21. Kühn CG: Claudii Galeni Opera Omnia Tomus XIII Lipsiae: Officina Libraria Car. Knoblochii 1827.

22. Pépin R: Quintus Serenus Sammonicus, Liber Medicinalis Paris: Presses Universitaires de France 1950

23. Cosmacini G, Gaudenzi G, Satolli R: Dizionario di storia della salute Torino: Einaudi 1996.

24. Accattatis L: Vocabolario del dialetto calabrese Castrovillari: Dai Tipi di Francesco Patitucci 1895

25. Marzano GB: Dizionario etimologico del dialetto calabrese Laureana di Borrello 1928.

26. De Giacomo G: Credenze e costumi dei villani di Cetraro. La Calabria 1893, V(7):49-51.

27. Capasso S, Grandolini G, Izzo A: Fitoterapia. Impiego razionale delle droghe vegetali Milano: Springer-Verlag Italia 2006

28. Schulz V, Hansel R, Tyler VE: Fitoterapia Razionale. Scienza e piante medicinali Fidenza: Mattioli 18852003

29. Conforti F, Menichini F, Loizzo MR, Statti AG, Rapisarda A, Menichini F, Houghton PJ: Antioxidant, a-amylase inhibitory and brine-shrimp toxicity studies on Centaurea centaurium L. methanolic root extract. Natural Product Research 2008, 22:1457-1466.

30. Saeedi M, Morteza-Semnani K: Chemical composition and antimicrobial activity of the essential oil of Heliotropium europaeum. Chemistry of Natural Compounds 2009, 45:98-99.

31. Arena A, Bisignano G, Pavone B, Tomaino A, Bonina FP, Saija A, Cristiani M, D'Arrigo M, Trombetta D: Antiviral and immunomodulatory effect of a lyophilized extract of Capparis spinosa L. buds. Phytoterapy Research 2008, 22:313-317.

32. Panico AM, Cardile V, Garufi F, Puglia C, Bonina F, Ronsisvalle S: Effect of hyaluronic acid and polysaccharides from Opuntia ficus indica (L.) cladodes on the metabolism of human chondrocyte cultures. $J$ of Ethnopharmacol 2007, 111:315-321.

33. Greige-Gerges H, Abou Khalil R, Abou Mansour E, Magdalou J, Chahine R, Ouaini N: Cucurbitacins from Ecballium elaterium juice increase the binding of bilirubin and ibuprofen to albumin in human plasma. Chemico-Biological Interactions 2007, 169:53-62.

34. Yarnell E, Abascal K, Hooper CG: Clinical Botanical Medicine Larchmont, NY: Mary Ann Liebert, Inc 2004

35. Foucault-Bertaud A, Lamy E, Senni K, Gaultier F, Ejeil AL, Piccirilli A, Piccardi N, Msika P, Godeau G, Gogly B: Protective effect of a vegetable extract from Lupinus albus (LU 105) on human gingival elastic fibers degradation by human leukocyte elastase. Clin Oral Invest 2003, 7:206-211.

36. Israili ZH, Lyoussi BA: Ethnopharmacology of the plants of genus Ajuga. Pak J Pharm Sci 2009, 22(4):425-462

37. Decreto del Ministero della Sanità della Repubblica Italiana: Gazzetta Ufficiale 181. Roma 1996

38. Sanyal AK, Bhattacharya SK: Arundo donax L. (Graminae). Phytichemical and pharmacological evaluation. Journal of Medical Chemistry 1969, 12:480-483.

39. Benigni R, Capra C, Cattorini PE: Piante medicinali. Chimica, Farmacologia e Terapia Milano: Inverni \& Della Beffa 1962.

40. Iorizzi M, Lanzotti V, De Marino S, Zollo F, Blanco-Molina M, Macho A, Munoz E: New Glycosides from Capsicum annuum L. Var. acuminatum. Isolation, Structure Determination, and Biological Activity. J Agric Food Chem 2001, 49:2022-2029.

41. Ksouri R, Falleh H, Megdiche W, Trabelsi N, Mhamdi B, Chaieb K, Bakrouf A Magné $C$, Abdelly $C$ : Antioxidant and antimicrobial activities of the edible medicinal halophyte Tamarix gallica L. and related polyphenolic constituents. Food and Chemical Toxicology 2009, 47:2083-2091.

42. Giachetti D, Taddei E, Taddei I: Diuretic and uricosuric activity of Parietaria judaica L. Bollettino della Società Italiana di Biologia Sperimentale 1986, 62:197-202.

43. Calvo MI: Anti-inflammatory and analgesic activity of the topical preparation of Verbena officinalis L. J of Ethnopharmacol 2006 107:380-382.

44. Vrchovská V, Spilková J, Valentão P, Sousa C, Andrade PB, Seabra RM: Antioxidative properties and phytochemical composition of Ballota nigra infusion. Food Chemistry 2007, 105:1396-1403.

45. Flamini G, Cioni PL, Puleio R, Morelli I, Panizzi L: Antimicrobial Activity of the Essential Oil of Calamintha nepeta and its Constituent Pulegone Against Bacteria and Fungi. Phytother Res 1999, 13:349-351.

46. Srinivasan K: Black Pepper and its Pungent Principle-Piperine: A Review of Diverse Physiological Effects. Critical Reviews in Food Science and Nutrition 2007, 47:735-748.

47. Mandalari G, Bennett RN, Bisignano G, Trombetta D, Saija A, Faulds CB, Gasson MJ, Narbad A: Antimicrobial activity of flavonoids extracted from bergamot (Citrus bergamia Risso) peel, a byproduct of the essential oil industry. Journal of Applied Microbiology 2007, 103:2056-2064.

48. Alzoreky NS, Nakahara K: Antibacterial activity of extracts from some edible plants commonly consumed in Asia. International Journal of Food Microbiology 2003, 80:223-230.

49. Passalacqua NG, Guarrera PM, De Fine G: Contribution to the knowledge of the folk plant medicine in Calabria region (Southern Italy). Fitoterapia 2007, 78:52-68.

50. Leporatti ML, Impieri M: Ethnobotanical notes about some uses of medicinal plants in Alto Tirreno Cosentino area (Calabria, Southern Italy). $J$ of Ethnobiol and Ethnomed 2007, 3:34-39.

51. Martino P: Atlante di piante officinali con antiche ricette di erboristeria contadina in Calabria Cosenza: Editoriale Progetto 20001992.

52. The Angiosperm Phylogeny Group guidelines. [http://www.mobot.org/ MOBOT/research/APWeb/].

53. Lombardi Satriani R: Ars salutaris. Rivista di Antropologia 1916, 21:265-269.

54. De Giacomo G: Usi e costumi di Cetraro. La Calabria 1892, V(3):19-21.

55. De Giacomo G: Il popolo di Calabria Castrovillari: Patitucci Editore 1896

56. Genovese F: La malaria in provincia di Reggio Calabria Firenze: Vallecchi Editore 1924.

57. De Giacomo G: Usi e costumi di Cetraro. La Calabria 1892, V(5):39-40

58. Pignatari Fl: Medicina popolare. Rimedi contro le febbri palustri. $\mathrm{La}$ Calabria 1895, VII(10):73-74

59. Pignatari Fl: Medicina popolare. L'aglio. La Calabria 1894, VI(12):90-91

60. Pignatari Fl: Medicina popolare. Rimedi contro le febbri palustri. $L a$ Calabria, Anno 1895, VII(11):81-82

61. Givigliano GP: Territorio e malaria nei Bruttii. Rivista storica italiana 2001, CXIII(3):583-613. 
62. Genovese F: La malaria nel "folk-lore" calabrese. La Propaganda Antimalarica 1912, 2:16-23.

63. Lanza B: Cassano. Rimedi popolari. // Regno delle due Sicilie descritto ed illustrato. 1853-1860 Calabria Ristampa anastatica. Napoli: Edizioni Paparo 2006, 13.

64. Geraci PO: La medicina del popolo di Calabria. Folklore della Calabria 1957, II(1):17-44.

65. Adriano A: Carmi, tradizioni, pregiudizi nella medicina popolare calabrese Cosenza: Tipografia Commerciale Antonio Pranno 1932.

66. Pagano L: Bisignano. Rimedi popolari e tradizionali. // Regno delle due Sicilie descritto ed illustrato. 1853-1860 Calabria Ristampa anastatica Napoli: Edizioni Paparo 2006, 80

67. Pignatari Fl: Medicina popolare - Una pagina originale di medicina popolare. La Calabria 1894, VII(4):26-27.

68. Marzano GB: Usi e costumi di Laureana di Borrello - Medicina Popolare. La Calabria 1890, III(4):29-30

69. Brancia V: Joppolo. Rimedi stravaganti. /l Regno delle due Sicilie descritto ed illustrato. 1853-1860 Calabria Ristampa anastatica Napoli: Edizioni Paparo 2006, 31.

70. Pagano L: Studi storici sulla Calabria. Natura economia storia in Calabria Napoli: 1892. Ristampa anastatica, Cosenza: Edizioni Cronaca di Calabria 1992.

71. Mollo S: Quadretti ed aspetti di vita folklorica calabrese Reggio Calabria: Tipografia Fata Morgana 1930.

72. De Marco D: (a cura di) La statistica del Regno di Napoli nel 1811 Ristampa anastatica, Roma: Accademia Nazionale dei Lincei 1988.

73. Tenore M: Memoria sul Pruno cocumiglia di Calabria: 15 dicembre 1827 Napoli. Reale Istituto d'Incoraggiamento alle Scienze Naturali 1828

74. Romeo V: La profilassi antimalarica in Calabria Reggio Calabria: Tipografia Panella 1946.

75. Pignatari FI: Medicina popolare - II pepe. La Calabria 1894, VII(3):18-19.

76. Galasso L: Arabi e beduini d'talia. Studi pratico-sociologici sul proletariato calabrese Polistena (RC): Tipografia Cristoforo Colombo 1915.

77. Corso R: Amuleti italiani con speciale riguardo a quelli calabresi. Calabria letteraria 1953, 7:8-9.

78. Donnarumma V: Cosenza Mariana Cosenza: Tipografia Chiappetta 1951

79. Iannicelli A: Paesi di Calabria. Magia, religiosità popolare e terapia empirica nella cultura subalterna in Calabria Castrovillari: II Coscile Editore 1991.

80. Heinrich M, Kufer J, Leonti M, Pardo-de-Santayana M: Ethnobotany and ethnopharmacology-Interdisciplinary links with the historical sciences. J of Ethnopharmacol 2006, 107:157-160.

81. De Natale A, Pezzatti GB, Pollio A: Extending the temporal context of ethnobotanical databases: the case study of the Campania region (southern Italy). J of Ethnobiol and Ethnomed 2009, 5:7-24.

82. De Martino E: Sud e Magia Milano: Feltrinelli 1959.

83. Pasquarelli MG: La malaria nelle credenze e pratiche popolari di Basilicata. Folklore 1922, VIII(3):154-168.

84. Turner NJ: The importance of a Rose: Evaluating the Cultural Significance of Plants in Thompson and Lillooet Interior Salish. Am Anthropol 1988, 90:272-290.

doi:10.1186/1746-4269-6-27

Cite this article as: Tagarelli et al:: Folk medicine used to heal malaria in Calabria (southern Italy). Journal of Ethnobiology and Ethnomedicine 2010 6:27.

\section{Submit your next manuscript to BioMed Central and take full advantage of:}

- Convenient online submission

- Thorough peer review

- No space constraints or color figure charges

- Immediate publication on acceptance

- Inclusion in PubMed, CAS, Scopus and Google Scholar

- Research which is freely available for redistribution 